Sädhanā, Vol. 12, Parts 1 \& 2, February 1988, pp. 1-14. (C) Printed in India.

\title{
Observations of supersonic free shear layers
}

\author{
D PAPAMOSCHOU and A ROSHKO \\ Department of Aeronautics, California Institute of Technology, \\ Pasadena, California 91125, USA
}

\begin{abstract}
Visual spreading rates of turbulent shear layers with at least one stream supersonic were measured using Schlieren photography. The experiments were done at a variety of Mach number-gas combinations. The spreading rates are correlated with a compressibility-effect parameter called the convective Mach number. It is found that for. supersonic values of the convective Mach number, the spreading rate is about one quarter that of an incompressible layer at the same velocity and density ratio. The results are compared with other experimental and theoretical results.
\end{abstract}

Keywords. Supersonic free shear layers; turbulent shear layers; convective Mach number; spreading rate.

\section{Introduction}

The supersonic turbulent free shear layer is fashionable again. The interest which developed in the 1960s was related mainly to the near wake problem; the present, renewed interest comes largely from problems in supersonic combustion. In the earlier period it had been noted that turbulent shear layers with supersonic velocity on one side and zero velocity on the other (e.g. at the edges of jets) spread more slowly than incompressible turbulent shear layers, and it was thought that the difference was attributable to the density differences that were present in the supersonic cases. Thus models incorporating Howarth-Darodnitsyn transformations, density-dependent eddy viscosities etc., were invented.

Whether density effects alone could account for the differences in spreading rate was the question that motivated the experiments of Roshko \& Brown $(1971,1974)$, who built an apparatus in which incompressible $(M=0)$ shear-layer flows with large density differences could be studied by simply using different gas combinations like nitrogen and helium, at low speeds. It was found that, while there is some effect of the density on spreading $\mathrm{r}, \mathrm{e}$, it is very much smaller than is observed in the supersonic case. The relative magnitudes are summarized in figure 15 of 
Roshko \& Brown (1974). For example, the shear layer spreading rate at the edge of a Mach 4 jet (with uniform total temperature) is about 0.4 that of an incompressible shear layer with the same ratio of densities. It must be concluded that compressibility, per se, plays an important role in the supersonic case. In fact, with the observation that subsonic turbulent shear layers contain large, so-called "coherent" structures reminiscent of those in the early stages of instability, and the realization that the turbulent shear layer growth is governed largely by its instability at the corresponding large scales, it became clear that these processes must be quite different in supersonic flow. In fact, there is visual evidence that large structures also exist in supersonic turbulent mixing layers (Ortwerth \& Shine 1977; Oertel 1979).

It has been found that some insights into turbulent growth rates can be obtained, at least qualitatively, from the amplification rates of instability waves in laminar shear layers (more precisely from stability calculations for typical shear-layer profiles of velocity and density). Even the simpler calculations which can be made on the instability of vortex sheets are useful. Some further discussion of the possible connection between shear-layer instability and turbulent growth rate is given below.

The discovery of organized large structure also led to the idea that the natural coordinate system in which to view the flow is one moving with those structures. In the instability analysis this coordinate system moves with the wave velocity; in turbulent flows the term "celerity" has been used by Favre et al (1967) to define the velocity of the moving frame in which the large structure is most nearly stationary (see Coles 1981). In this convecting frame then, the Mach number $M_{c}$, which is called the "convective" Mach number, will be different from that in the laboratory system. It may possibly be less than unity even when there is supersonic flow in the laboratory system. It would also be appropriate to call it the "intrinsic" Mach number, since it is the one that is relevant for Mach number effects.

In practically all the available data on supersonic shear layers so far the low speed side was at rest $\left(M_{2}=0\right)$. Exceptions, to our knowledge, are experiments done by Demetriades \& Bower (1982), Sanderson \& Steel (1970) and Ortwerth \& Shine (1977). Demetriades \& Bower's results apply for the laminar-transitional regime and therefore cannot be used for predicting turbulent shear layer behaviour. Sanderson \& Steel's results are hard to interpret and their shear layers are mostly wake-dominated. In Ortwerth \& Shine's experiment a stream of helium at $M=3$ mixes with a stream of nitrogen also at $M=3$. A spark Schlieren photograph shows a slowly growing mixing layer with large scale structure.

One of the main purposes of the experiment described here was to examine the effects of varying $M_{2}$ from subsonic to supersonic values while keeping $M_{1}>1$. This would provide a useful test of the intrinsic Mach number concept. In addition, we wanted to study the effects of a larger variation of the parameter $\rho_{2} / \rho_{1}$ than that afforded by the typical jet experiment of a single gas and with all total temperatures at room values. Accordingly, we designed and built an apparatus, essentially a variation of the Roshko \& Brown (1974) apparatus, in which two streams of gases are allowed to mix downstream of a splitter plate.

In this work we present the mixing layer spreading rates, obtained experimentally with instantaneous Schlieren photographs, at a variety of Mach number-gas combinations. We assume that the Reynolds numbers are high enough to assure 
that the mixing layers are fully developed turbulent everywhere except in a very small region near the splitter plate. Our goal is to examine the effect of compressibility on the spreading rates and to determine what are the fundamental parameters that describe this effect.

The following table lists typical experimental conditions in which the mixing layers were observed:

Mach number range

0 to 4

Density ratio range

0.2 to 10

Typical unit Reynolds number

$10^{5} \mathrm{~cm}^{-1}$

Typical test section pressure

1 psia (7000) $\mathrm{Pa})$

Gases used

nitrogen, helium, argon

\section{Experimental facility}

\subsection{Supersonic mixing apparatus}

A schematic of the apparatus is shown in figure 1. It is essentially a two-stream blow-down supersonic wind tunnel with two independent supply sides. Each supply side is connected to pressure regulated gas cylinders. The downstream side is connected to a low pressure tank which is evacuated by a vacuum pump.

After passing through flow management devices, each gas is expanded to a design Mach number by means of an appropriately contoured centrebody, downstream of which the two gases mix. The centrebody contours, calculated by the method of characteristics, are designed to produce uniform, wavefree flows on each side. The centrebody is replaceable, allowing the use of a number of centrebodies for various Mach number combinations.

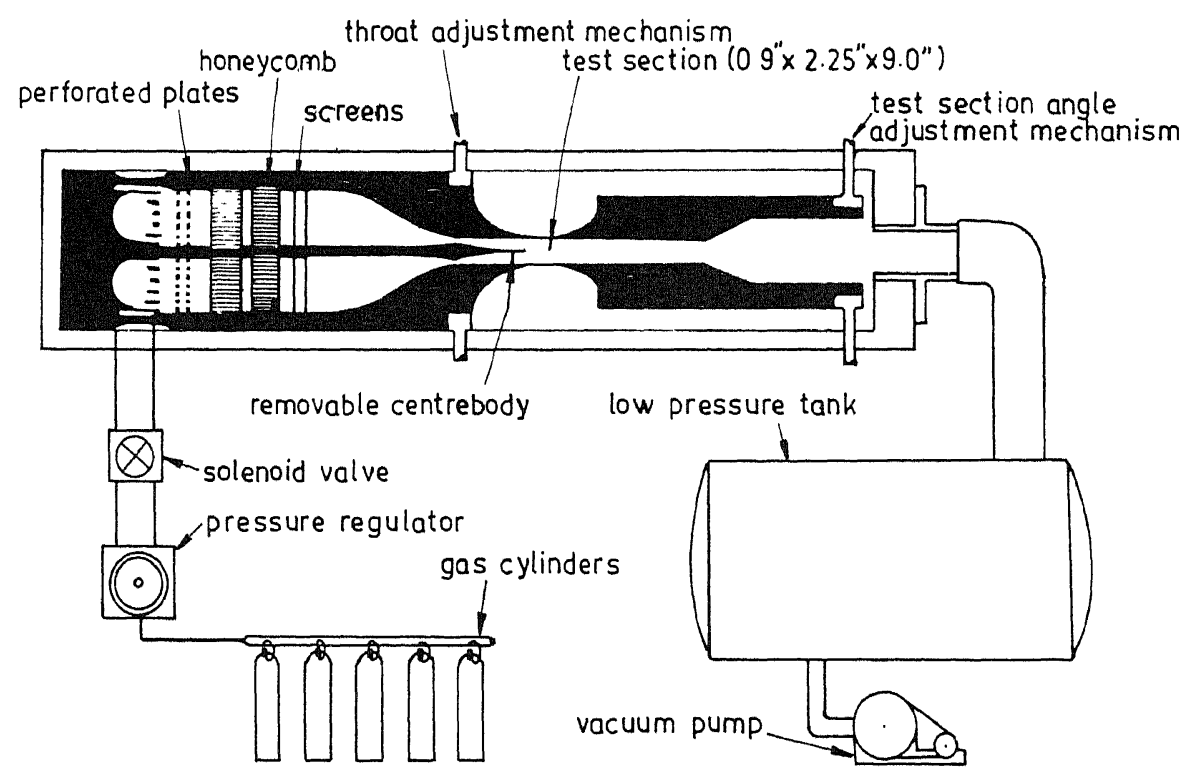

Figure 1. Experimental facility. 
The test section is $2.3 \mathrm{~cm}(0.9$ in.) high, $5.7 \mathrm{~cm}(2.25$ in.) wide and $23 \mathrm{~cm}(9$ in. $)$ long. Optical quality glass windows extend from upstream of the centrebody tip to the end of the test section. The angle of each test section wall is adjustable within $\pm 3^{\circ}$ to compensate for possible displacement thickness effects. The nozzle throat heights are also adjustable to ensure proper use of each centrebody.

The operation of the facility is intermittent, each run lasting typically 1.5 to $2 \mathrm{~s}$. The maximum limit on total pressures is 100 psig. The total temperatures at the beginning of each run are $300 \mathrm{~K}$ with a drop of 5 to $10 \mathrm{~K}$ occurring during each run due to expansion in the gas cylinders.

\subsection{Schlieren system}

The Schlieren system used is a conventional one with instantaneous or continuous illumination. The knife edge is horizontal and the slit in front of the light source has an adjustable opening for varying the sensitivity of the system.

The instantaneous illumination is provided by a spark source of 20 nanosecond duration (Xenon Corporation Nanolamp Model N-787B). A continuous source with a 100 Watt tungsten halogen lamp (Oriel Model 6322) is used for adjustment. The parallel beam entering the test section has a diameter of $10 \cdot 1 \mathrm{~cm}$ (4 in.). The Schlieren system is stationary but the flow channel can be traversed on rails, to allow photography of all parts of the test section.

\subsection{Pressure and temperature measurement}

Static pressure ports along the test section and total pressure ports are connected to pressure transducers (Setra Systems Model 204) through a Scanivalve system. The output of the transducers is recorded on a Nicolet digital oscilloscope and stored on discs. Two total temperature thermocouples are connected to an electronic thermometer (Omega Model $115 \mathrm{TC}$ ) whose output is recorded on a plotter.

\subsection{Control of the experiment}

The starting and stopping of the flow, photography and recording are done automatically with an Intel 8085 microprocessor connected to a system of relays. Each Schlieren picture is accompanied by a record of all relevant pressures and temperatures.

\section{Concept of convective Mach number}

\subsection{Definition of convective Mach number}

In investigating the effects of Mach number on the stability and mixing of compressible shear layers (figure 2) one would like to find parameters which will correlate and unify the results for various flow conditions. In shear layers where $M_{2}=0, M_{1}$ itself is such a parameter (for given total conditions). However, in shear layers with $M_{2} \neq 0$ and $a_{2} \neq a_{1}$ the choice of the right parameters is not
clear.

Some insight into the problem can be gained by first treating simple flows, like the unstable temporally developing vortex sheet in compressible flow. In the case of the vortex sheet it is known that the important parameters are 


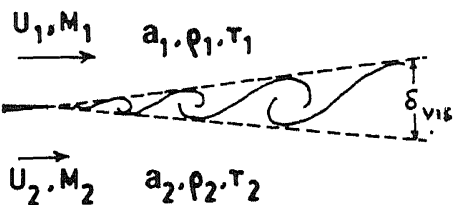

Figure 2. Shear layer nomenclature.

$$
M_{c_{1}}=\left(U_{1}-c_{r}\right) / a_{1}
$$

and

$$
M_{c_{2}}=\left(c_{r}-U_{2}\right) / a_{2}
$$

where $c_{r}$ is the real phase speed corresponding to neutral $(c=0)$ disturbances. The requirement of continuity of pressure at the interface of the two gases is the boundary condition which determines $c_{r}$; using Ackeret's (1927) linearized theory for the pressure perturbations this results in

$$
\gamma_{1} M_{c_{1}}^{2} /\left(\left|M_{c_{1}}^{2}-1\right|\right)^{1 / 2}=\gamma_{2} M_{c_{2}}^{2} /\left(\left|M_{c_{2}}^{2}-1\right|\right)^{1 / 2}
$$

With the definitions of $M_{c_{1}}$ and $M_{c_{2}}$, this equation implies the solution for $c_{r}=c_{r}\left(U_{1}, U_{2}, a_{1}, a_{2}, \gamma_{1}, \gamma_{2}\right)$ and thus the values of $M_{c_{1}}$ and $M_{c_{2}}$. Since we are only dealing with monatomic and diatomic gases, $M_{c_{1}}$ and $M_{c_{2}}$ are equal or very close.

The importance of $M_{c_{1}}$ as a stability determining parameter arises from the fact that when $M_{c_{1}}$ exceeds a critical value only neutral disturbances exist and the vortex sheet is stable (Miles 1958).

For turbulent free shear layers a similar approach can be taken by defining

$$
M_{c_{1}}=\left(U_{1}-U_{c}\right) / a_{1} \text { and } M_{c_{2}}=\left(U_{c}-U_{2}\right) / a_{2}
$$

where $U_{c}$ is now the convective velocity of dominant waves or structures. The convective velocity might again be calculated from (1), i.e. by requiring static pressure equality and calculating it in a coordinate system moving with the structures. There are two objections to this: first, we are no longer dealing with a vortex-sheet interface so there is really no way to specify static pressure equality; second, one might prefer not to use linearization as was done for (1). An alternative, related approach has been proposed by several investigators. It was first suggested to us by Dimotakis (private communication, and 1984) and is implicit in Coles (1981) sketches of streamlines in the moving coordinates of the large structures (figure 3 ). In this coordinate system there is a saddle point between the structures; it is a common stagnation point for both streams, thus implying equality of total pressures in the two streams in that system. For equal static pressures this results in

$$
\left\{1+\left[\left(\gamma_{1}-1\right) / 2\right] M_{c_{1}}^{2}\right\}^{\gamma_{1} /\left(\gamma_{1}-1\right)}=\left\{1+\left[\left(\gamma_{2}-1\right) / 2\right] M_{c_{2}}^{2}\right\}^{\gamma_{2} /\left(\gamma_{2}-1\right)} \text {. }
$$

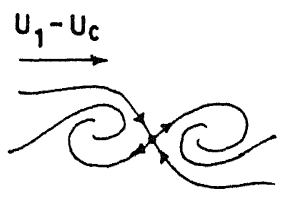

$\widehat{u_{c}-U_{2}}$ Figure 3. Shear layer structure in convecting reference (after Coles 1981). 
This result has been obtained by Bogdanoff (1982) using a slightly different argument. It should also be noted that Oertel $(1979,1982)$ has obtained excellent experimental evidence supporting the concept of large coherent structures and associated convective velocities in supersonic mixing layers.

For $M_{c_{1}}$ and $M_{c_{2}}$ which are not very large, (2) can be approximated by its subsonic version:

$$
\frac{1}{2} \rho_{1}\left(U_{1}-U_{c}\right)^{2}=\frac{1}{2} \rho_{2}\left(U_{c}-U_{2}\right)^{2}
$$

which gives

$$
U_{c} / U_{1}=\left(1+r s^{\frac{1}{2}}\right) /\left(1+s^{\frac{1}{2}}\right) .
$$

Equation (4) together with the definition of convective Mach numbers gives the following relation:

$$
M_{c_{2}}=\left(\gamma_{1} / \gamma_{2}\right)^{\frac{1}{2}} M_{c_{1}}
$$

which can also be obtained directly from (1) for small $M_{c_{1}}$ and $M_{c_{2}}$. As for the vortex sheet, $M_{c_{1}}=M_{c_{2}}$ when $\gamma_{1}=\gamma_{2}$. From the definition of convective Mach numbers we then get the following expression for $U_{c}$ :

$$
U_{c}=\left(a_{2} u_{1}+a_{1} u_{2}\right) /\left(a_{1}+a_{2}\right), \text { for } \gamma_{2}=\gamma_{1},
$$

which has the form of a speed-of-sound weighted average.

$M_{c_{1}}$ and $M_{c_{2}}$ are slightly different when $\gamma_{1}=7 / 5$ and $\gamma_{2}=5 / 3$ or the reverse. Figure 4 shows the dependence of $M_{c_{1}}$ on $M_{1}$ and $M_{2}$ for various gas combinations. $M_{c_{1}}$ has been calculated using its definition and (3) for uniform total temperature. The error that results from using (3) instead of (2) is very small for the range of $M_{c_{1}}$ plotted on the figure.

\subsection{Effect of $M_{c}$ on stability of vortex sheet}

To illustrate the effect of convective Mach number on the stability of a vortex sheet the following simplified argument is presented. In a frame of reference moving with the phase speed $c_{r}$ (figure 5a) we replace the interface of the two gases with a thin flexible membrane (figures $5 \mathrm{~b}, \mathrm{c}$ ). Then the problem reduces to that of flow past a wave-shaped boundary, first treated by Ackeret (1927). For $M_{c_{1}}$ and $M_{c_{2}}$ greater than 1 , the pressures on both sides of the membrane balance everywhere and thus the membrane is neutrally stable (figure $5 b$ ). When at least one of the convective Mach numbers is less than 1, the pressure on both sides of the membrane cannot be balanced and thus the membrane is unstable (figure $5 \mathrm{c}$ ).

The above argument can be used only descriptively to explain the vortex sheet stability, the analysis being quite different (Miles 1958). The basic result, however, is similar in the sense that there is a convective Mach number beyond which the vortex sheet is neutrally stable. For equal specific heat ratios and speeds of sound, the vortex sheet is stable for $M_{c_{1}}>2^{1 / 2}$. 

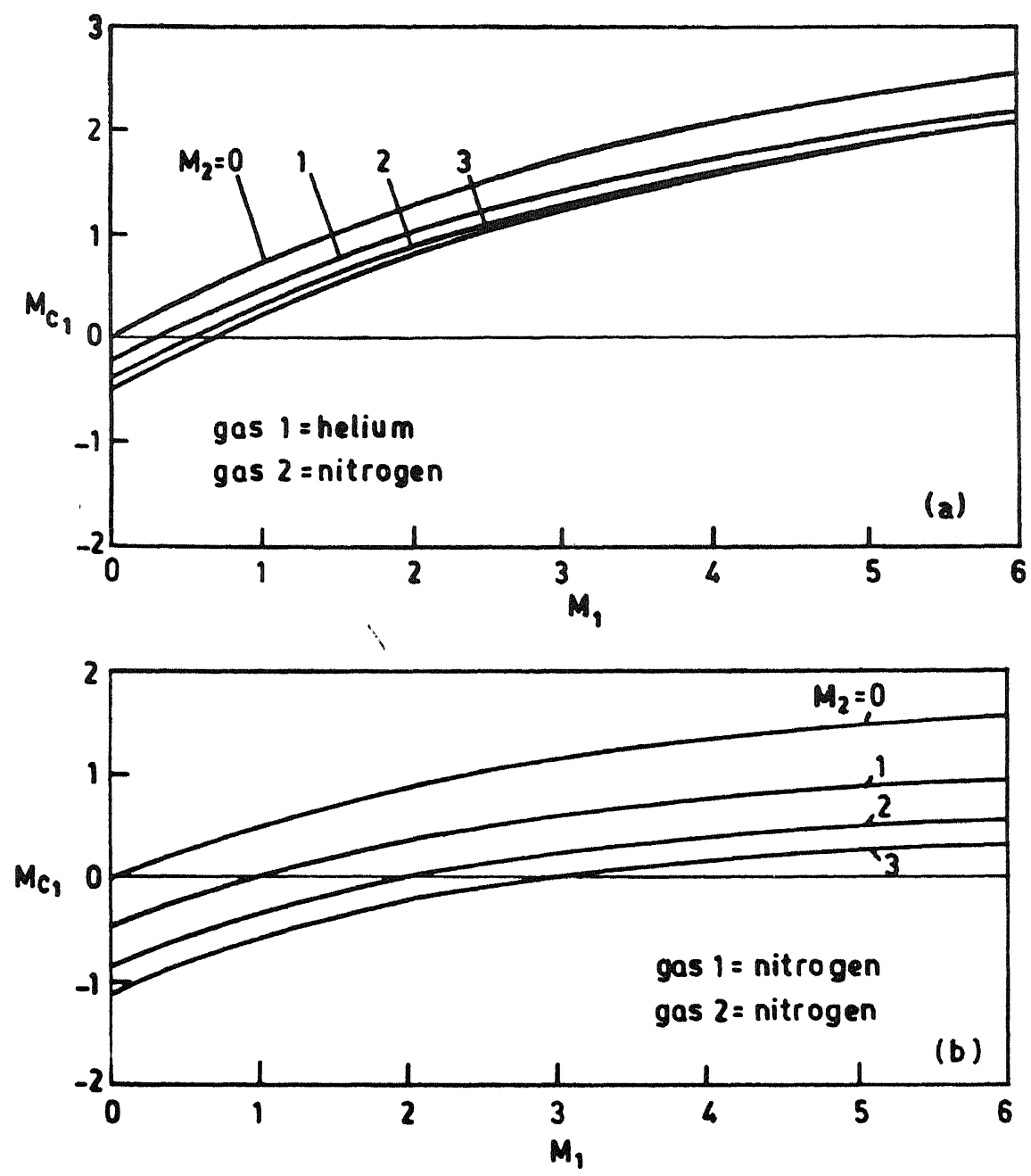

Figure 4. Convective Mach number for $\mathrm{N}_{2}-\mathrm{N}_{2}$ and $\mathrm{He}-\mathrm{N}_{2}$ combinations.

(a) $-\cdots \frac{U_{1}-c_{r_{1}} M c_{1}}{\frac{c_{r}-U_{2} \cdot M_{c_{2}}}{c_{1}}}$

(b) $\frac{m_{c_{1}>1}}{m_{c_{2}>1}}+1$

(c) $\frac{M_{c_{1}<1}+1}{M_{c_{2}<1}+1}+1$

Figure 5. Vortex sheet stability argument. 


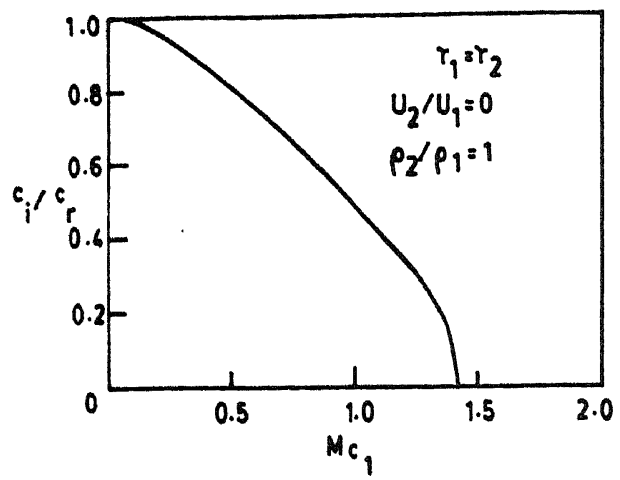

Figure 6. Vortex sheet amplification rate.

The reduction in amplification rate with $M_{c_{1}}=M_{c_{2}}$ is shown in figure 6 for $\gamma_{1}=\gamma_{2}, r=0$ and $s=1$. This is the result of the temporal analysis that has been transformed to the spatial growth rate $c_{i} / c_{r}$, the wavenumber being chosen to be unity. The growth rate is normalized to be 1 at $M_{c_{1}}=0$.

\subsection{Effect of $M_{c}$ on shear layers of finite thickness}

In the case of the vortex sheet we can always find a set of conditions for which the freestream velocity is supersonic relative to the disturbance velocity. This is not true in shear layers of finite thickness where, no matter how large $M_{c}$ is, there exists always a flow region where the local velocity is subsonic relative to the disturbance velocity. Disturbances in this region might amplify and lead to turbulent growth of the shear layer. This is reflected in the calculations of Blumen et al (1975), where it is shown that there is instability of two-dimensional disturbances at all values of $M_{c}$.

Gropengiesser (1970) has computed maximum amplification rates $\left(-\alpha_{i}\right)$ for spatially developing compressible shear layers with a Lock velocity profile. One of his results is shown in figure 7 where $-\alpha_{i}$ is plotted here against $M_{c_{1}}\left(=M_{c_{2}}\right)$ for $\gamma_{2}=\gamma_{1}, r=0$ and $s=1$ and is normalized to be 1 at $M_{c_{1}}=0$. Notable are the drastic decrease of $-\alpha_{i}$ with increasing $M_{c_{1}}$ and the flattening of the $-\alpha_{i}$ versus $M_{c_{1}}$ curve for $M_{c_{1}}>1$.

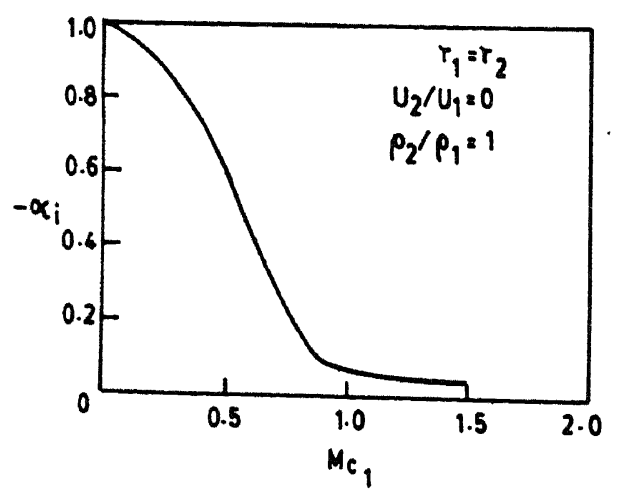

Figure 7. Shear layer amplification rate as computed by Gropengiesser (1970). 


\section{Results and discussion}

\subsection{Schlieren photography}

Instantaneous Schlieren photographs for a variety of flow conditions are shown in figure 8 . Each picture covers a 4-inch length of the test section. Two pictures are shown for each condition, the second one downstream of the first, with a $1 / 2$ inch overlap unless otherwise indicated. The quality of the pictures depends on the refractive index gradients. These gradients decrease as the mixing region thickens, therefore it was usually necessary to increase the Schlieren sensitivity in order to visualize the downstream pictures. Visualization was most difficult for the case with large growth rates and small refractive index differences. It should be noted that due to the low test section pressures the refractive indices are very small and visualization was possible only with the knife edge horizontal.

\subsection{Measurement of growth rates}

Visual growth rate is measured by drawing straight-line mean tangents to the edges of the shear layer, as done by Roshko \& Brown (1974). Considering the smallness of the growth rates and in some cases the uncertainty in defining the edges of the layer, such procedure is subjective and the error may be up to $\pm 20 \%$. For each flow condition, the growth rate presented here is the average of those in several pictures at the same condition. In each case the Reynolds number is the highest possible to assure that the flow is fully developed.

\subsection{Comparison with incompressible growth rates}

In trying to uncouple the effects of $M_{c}$ from the other flow parameters, we must compare the compressible growth rate to the incompressible one at the same velocity and density ratio. It is important then to establish a relation by which we can accurately estimate the effects of velocity and density ratio on the growth of incompressible shear layers.

In establishing such a relation, we assume that the main effect of density ratio is to determine the convective velocity $U_{c}$ as given by (4). In a frame of reference moving with $U_{c}$ the density ratio then drops out of the picture and we arrive at the following relation by dimensional analysis,

$$
\delta / x \sim \Delta U / U_{c}
$$

For visual thickness this becomes,

$$
\delta_{\text {vis }} / x=0.17 \Delta U / U_{c}=(1-r)\left(1+s^{\frac{1}{2}}\right) /\left(1+r s^{\frac{1}{2}}\right) .
$$

This relation has been proposed by Bogdanoff (1984) and other similar ones have been proposed by Brown (1974) and Dimotakis (1984). Figure 9 shows that (8) agrees quite well with experimental data.

The effect of $M_{c}$ can now be shown by forming the ratio $\delta^{\prime} / \delta_{i}^{\prime}$, where $\delta^{\prime}=\mathrm{d} \delta / \mathrm{d} x$ is the actual growth rate and $\delta_{i}^{\prime}$ is the incompressible growth rate at the same values of $r$ and $s$, and plotting it against $M$. This is done in figure 10 for the growth rates measured in the present experiments. In figure 11, the results of other experiments, in all of which $M_{2}=0$ and both gases are air, are plotted in the same manner. 


\subsection{Discussion}

The drastic growth rate reduction with increasing $M_{c}$ is evident in the experimental results presented here. It is clear that this reduction is not due to density effects, as previously believed, but to the stabilizing effect of $M_{c}$. It is worth noting that this
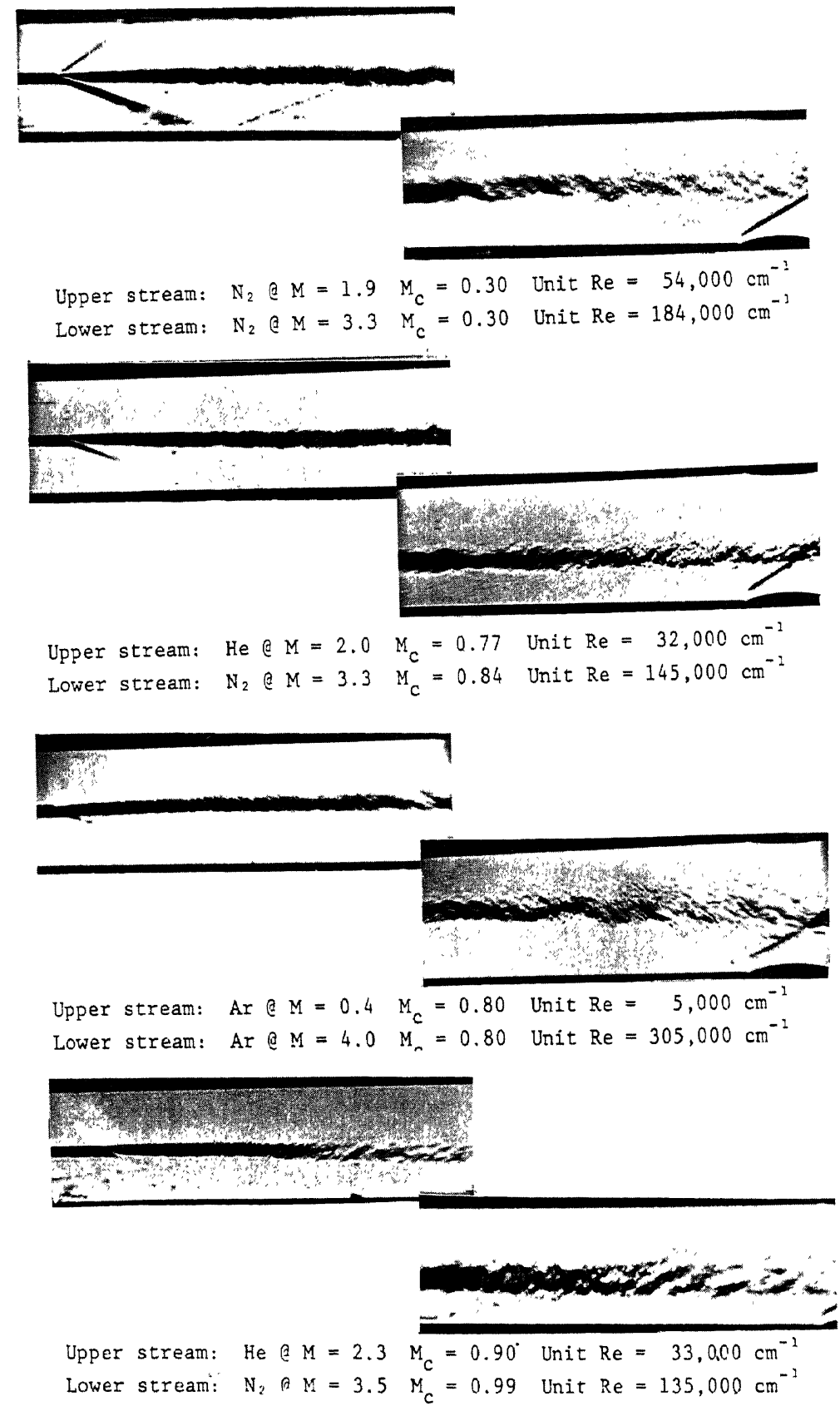

Figure 8 (For caption see facing page.) 


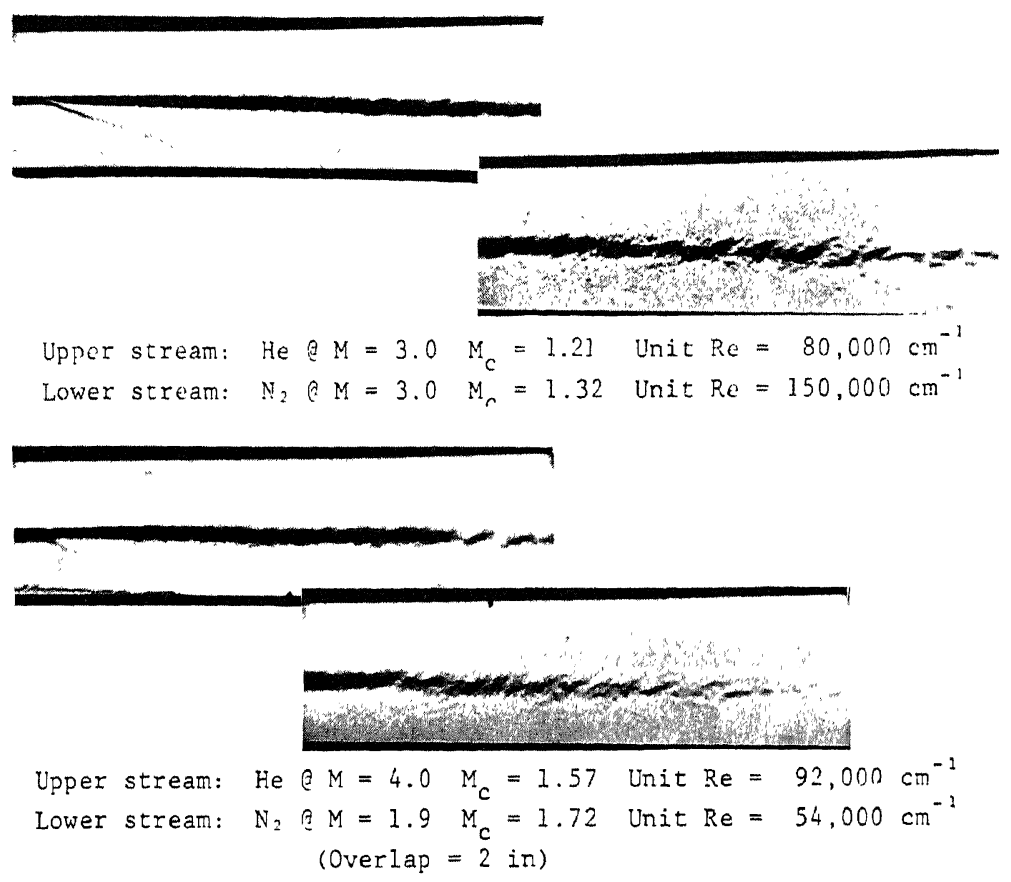

Figure 8. Schlieren photographs for a variety of flow conditions.

reduction levels off as $M_{c}$ exceeds 1 , something that appears in the present results and in those of other experiments. The amplification rates computed by Gropengiesser (1970) have the same trends as the experimental results for growth rates.

In the present experiments, the growth rate reduction for $M_{c}>1(\approx 75 \%)$ is considerably larger than that in the other experiments $(\approx 50 \%)$. One explanation for this difference may be the fact that in the other experiments with $M_{2}=0$ upstream propagation of disturbances is possible. Such upstream coupling might enhance the growth rate; it would also tend to be facility dependent. More likely, the difference may also arise from the fact that in the present experiment the

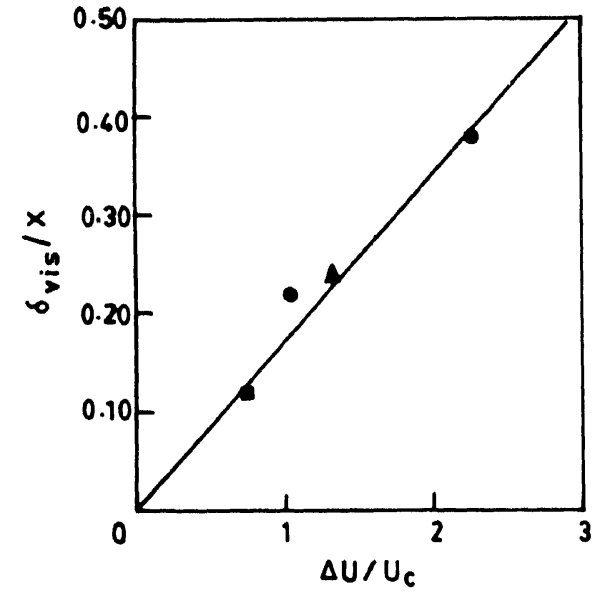

Figure 9. Visual thickness of incompressible shear layer. and data points from Roshko \& Brown (1974) for $s=7$ and $s=1 / 7$, respectively; $\Delta$ from Dimotakis \& Brown (1976) $(s=1)$. 


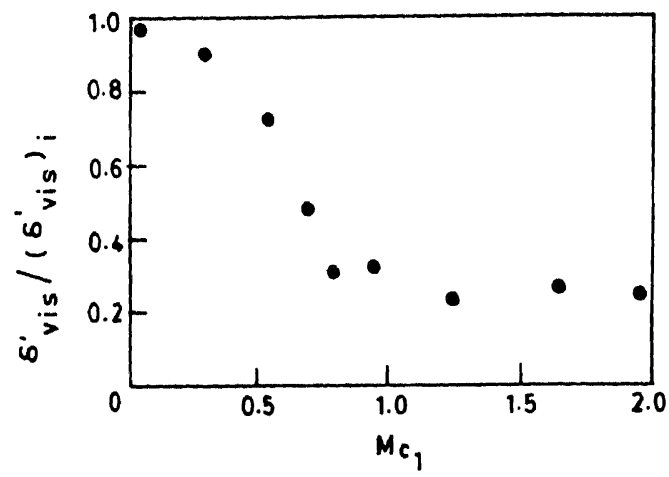

Figure 10. Present experimental values of visual thickness.

comparison is based on visual thickness measured from photographs, while in the other experiments it is the vorticity thickness, $\delta_{\omega}$, based on mean velocity profiles, which is compared. The ratio $\delta_{\text {vis }} / \delta_{\omega}$ may be a function of $M_{c}$; the above results would then suggest that this ratio decreases for $M_{c}>1$.

In forming the ratio $\delta^{\prime} / \delta$ we hope to have uncoupled the effects of $M_{c}$ from those of $r$ and $s$. While this may be true to first order, the effectiveness of $M_{c}$ in reducing the growth rate may still depend on $r$ and $s$. Furthermore, all the above discussion of shear layer instability has been limited to two-dimensional disturbances. It has been pointed out by several investigators (Lessen et al 1965, for example) that three-dimensional (oblique) disturbances can exist in supersonic shear layers. The effect of oblique waves on the vortex sheet stability can be accounted for by replacing $M_{c}$ by an effective convective Mach number $M_{c} \cdot \cos \theta$ where $\theta$ is the angle between wave propagation and flow direction. Bogdanoff (1982) suggests that the above transformation can be used to determine an effective $M_{c}$ in shear layers of finite thickness. We therefore expect the shear layer to become more unstable with increased obliquity of the disturbances. The current experimental set-up was not suitable for observing the possible presence of oblique waves in the flow, so nothing definite can be said about their effect on the present results. The consistent inhibition of growth rate for $M_{c}>1$ may indicate that if there exist three-dimensional waves, their obliquity is small.

With our present limited measurements the results are suggestive rather than definitive. To improve this, we are planning to measure Pitot-pressure and total-temperature profiles across the shear layer.

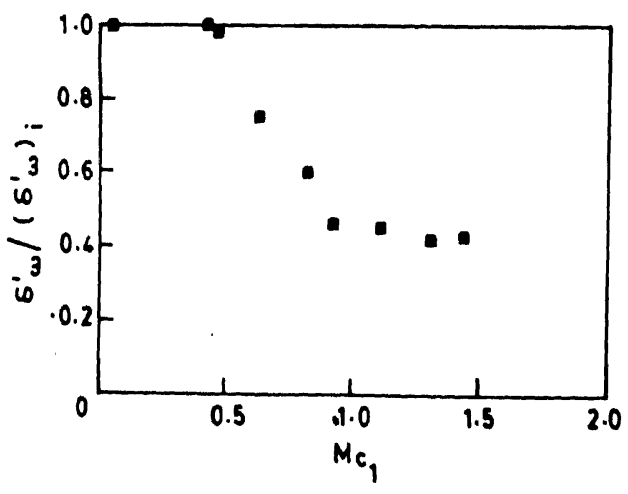

Figure 11. Experimental values of vorticity thickness obtained by other investigators as compiled in figure 1 of Bogdanoff (1982). 


\section{Concluding remarks}

The good correlation of experimentally obtained growth rates with $M_{c}$ at a variety of Mach numbers, density ratios and velocity ratios supports the idea that the convective Mach number is useful as a compressibility-effect parameter. It is found that the compressibility effect becomes significant at about $M_{c}=0.5$, increasing rapidly, then tending to level off for $M_{c}>1$. The compressibility effect on turbulent growth rate is mainly an effect on the stability of the flow, rather than a density effect, as indicated by the corresponding effect on amplification rates of waves in vortex sheets or laminar free shear layers. The overall reduction in growth rate for $M_{c}>1$ is by a factor of about 2 for vorticity thickness and about 4 for visual thickness. Thus the ratio of visual thickness/vorticity thickness appears to decrease by about a factor of 2 . More work is needed to refine these results and, especially, to establish quantitatively how mixing rates (molecular, chemical) decrease with increasing $M_{c}$.

This work is being supported by a research grant from the Rockwell International Corporation Trust.

\section{List of symbols}

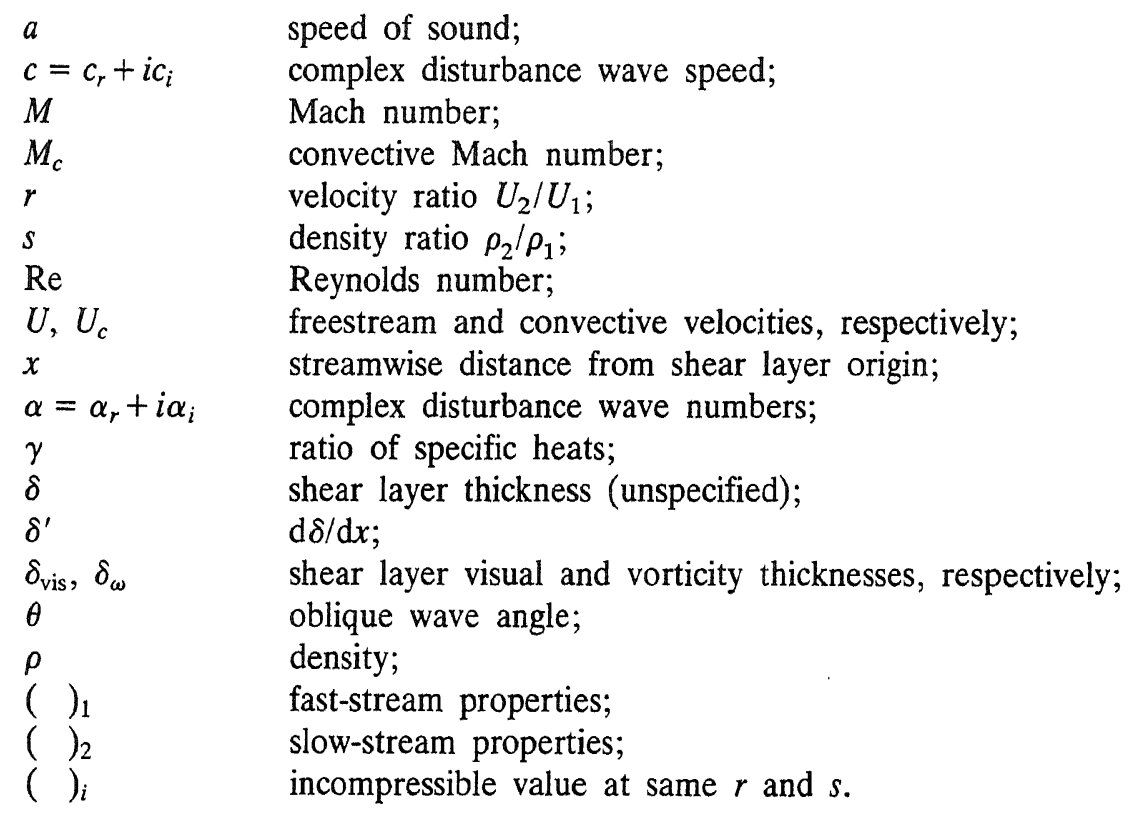

\section{References}

Blumen W, Drazin P G, Billings D F 1975 J. Fluid Mech. 71: 305-316

Bogdanoff D W $1982^{\circ}$ AIAA J. 21: 926-927 
Bogdanoff D W 1984 AlAA J. 22: 1550-1555

Brown G L 1974 Fifth Australasian conference on hydraulics and fluid mechanics (New Zealand: Christchurch) pp. 352-359

Coles D 1981 Proc. Indian Acad. Sci. (Eng. Sci.) 4: 111-127

Demetriades A, Bower T L 1982 Experimental study of transition in a compressible free shear layer, AFOSR-TR No. 83-0144

Dimotakis P E 1984 Entrainment into a fully developed, two-dimensional shear layer, AIAA-84-0368 Dimotakis P E, Brown G L 1976 J. Fluid Mech. 78: 535-560

Gropengiesser H 1970 Study of the stability of boundary layers and compressible fluids, NASA TT-F-12,786

Lessen M, Fox J A, Zien H M 1965 J. Fluid Mech. 23: 355-367

Miles J W $1958 \mathrm{~J}$. Fluid Mech. 4: 538-552

Oertel H 1979 Proc. 12th Int. Symp. of Shock Tubes and Waves, Jerusalem pp. 266-275

Oertel H 1982 Structure of complex turbulent shear flow, IUTAM Symp. Marseille

Ortwerth P J, Shine A J 1977 On the scaling of plane turbulent shear layers, AFWL-TR-77-118

Roshko A, Brown G L 1971 The effect of density difference on the turbulent mixing layer, AGARD CP-93

Roshko A, Brown G L 1974 J. Fluid Mech. 64: 775-781

Sanderson R J, Steel P C 1970 Results of experimental and analytical investigations in compressible turbulent mixing, Martin Marietta, R-70-48638-003 